\title{
Association of lipoprotein(a) with lower extremity artery disease and cardiovascular outcomes after peripheral revascularization
}

\begin{tabular}{|c|c|}
\hline & $\begin{array}{l}\text { National Medical Research Center of Cardiology } \\
\text { Ministry of Health of the Russian Federation, Moscow } \\
\text { 3rd Cherepkovskaya street, 15a, Moscow 121552, Russian Federation } \\
\text { ntmoyan@gmail.com, marat ezhov@mail.ru }\end{array}$ \\
\hline \multicolumn{2}{|c|}{ Narek Tmoyan, Marat Ezhov, Elena Klesareva, Marina Afanasieva, Olga Afanasieva, Sergei Pokrovsky } \\
\hline AM & $\begin{array}{l}\text { To evaluate the relationship of lipoprotein }(a)[L p(a)] \text { level with lower extremity artery disease (LEAD) and } \\
\text { cardiovascular outcomes after lower extremity revascularization. }\end{array}$ \\
\hline $\begin{array}{l}\text { MATERIAL } \\
\text { AND } \\
\text { METHODS }\end{array}$ & $\begin{array}{l}\text { The study included } 284 \text { patients with LEAD and } 338 \text { patients without significant atherosclerosis of coronary, } \\
\text { carotid and lower limbs arteries (control group). Of } 284 \text { patients with LEAD, } 111 \text { underwent lower extremity } \\
\text { revascularization. Lp(a) concentration was determined by our home ELISA. Atherosclerotic lesions of lower limb } \\
\text { arteries were assessed with ultrasound and/or angiography. LEAD is defined by the presence of severe }(\geq 50 \%) \\
\text { atherosclerotic stenosis and ankle-brachial index }<0.9 \text {. Coronary heart disease was diagnosed in } 73 \% \text { of patients } \\
\text { with LEAD and severe carotid atherosclerosis in } 60 \% \text {. The results are given as means and standard deviations, } \\
\text { medians and interquartile ranges, or numbers (percentages). Mann-Whitney U test was used while comparing the } \\
\text { quantitative characteristics of two groups. In order to compare frequency data between groups Fisher's exact test } \\
\text { was used. The cardiovascular outcomes were recurrent intermittent claudication, limb amputation, stroke, } \\
\text { transitory ischaemic attack, non-fatal myocardial infarction, unstable angina, repeated revascularization, } \\
\text { cardiovascular death. }\end{array}$ \\
\hline
\end{tabular}

\section{RESULTS}

Table 1. Baseline characteristics of study groups$$
\begin{array}{|l|}
\hline \\
\hline \mathrm{Ag} \\
\hline \mathrm{M} \\
\hline \mathrm{H} \\
\hline \mathrm{S} \\
\hline \text { Typ } \\
\hline \text { To } \\
\hline \text { Tr } \\
\hline \text { HD } \\
\hline \text { LD } \\
\hline \text { LD } \\
\hline \text { St } \\
\hline
\end{array}
$$

\begin{tabular}{|c|c|c|}
\hline & \multicolumn{2}{|c|}{$n=622$} \\
\hline & $\begin{array}{l}\text { LEAD } \\
n=285\end{array}$ & $\begin{array}{c}\text { Control } \\
n=337\end{array}$ \\
\hline Age, years & $69 \pm 9$ * & $56 \pm 12$ \\
\hline Male gender, n (\%) & $236(83 \%) *$ & $150(45 \%)$ \\
\hline Hypertension, n (\%) & $242(85 \%) *$ & $195(58 \%)$ \\
\hline Smoking, n (\%) & $175(61 \%) *$ & $94(28 \%)$ \\
\hline Type 2 diabetes, $\mathrm{n}(\%)$ & $81(28 \%) *$ & $49(15 \%)$ \\
\hline Total cholesterol, mmol/l & $5.0 \pm 1.4$ * & $6.1 \pm 1.5$ \\
\hline Triglycerides, mmol/l & $1.8 \pm 1.2 *$ & $2.0 \pm 1.0$ \\
\hline HDL-cholesterol, mmol/l & $1.2 \pm 0.4$ * & $1.3 \pm 0.4$ \\
\hline LDL-cholesterol, mmol/l & $2.9 \pm 1.2$ * & $3.9 \pm 1.3$ \\
\hline LDL-cholesterol corr, mmol// & $2.5 \pm 1.1 *$ & $3.7 \pm 1.3$ \\
\hline Statin therapy, n (\%) & $258(91 \%) *$ & $78(23 \%)$ \\
\hline
\end{tabular}

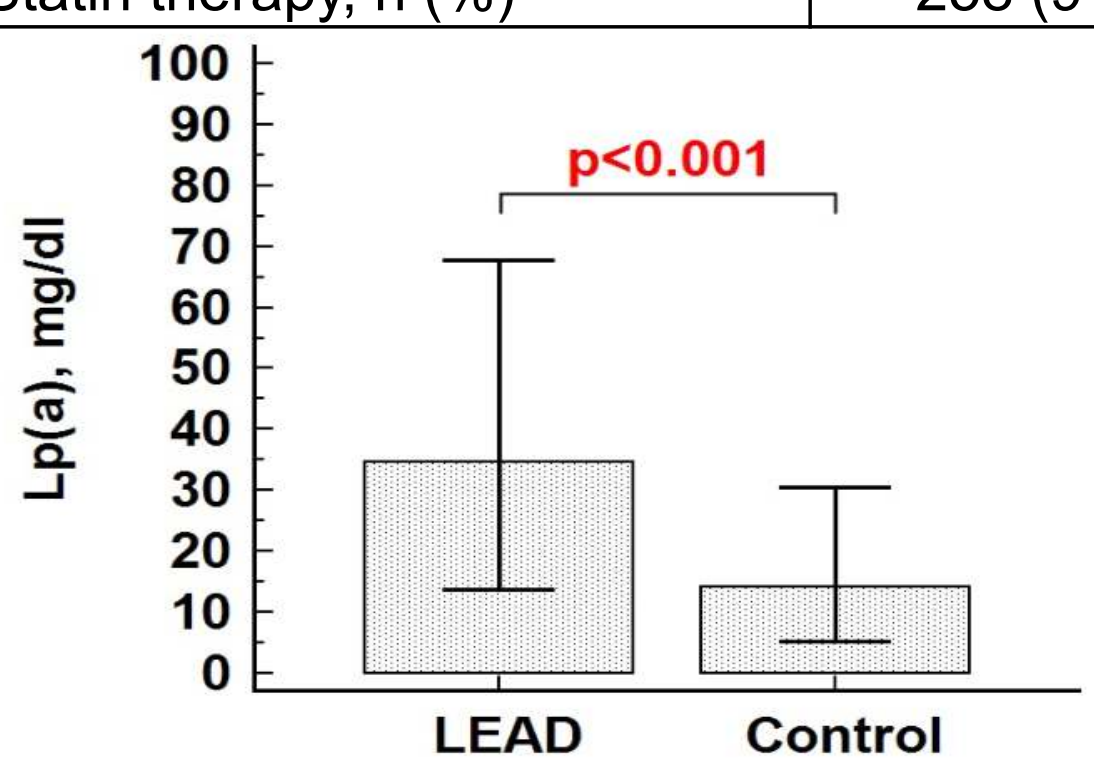

Figure 2. Relationship of $L p(a)$ level with LEAD

क

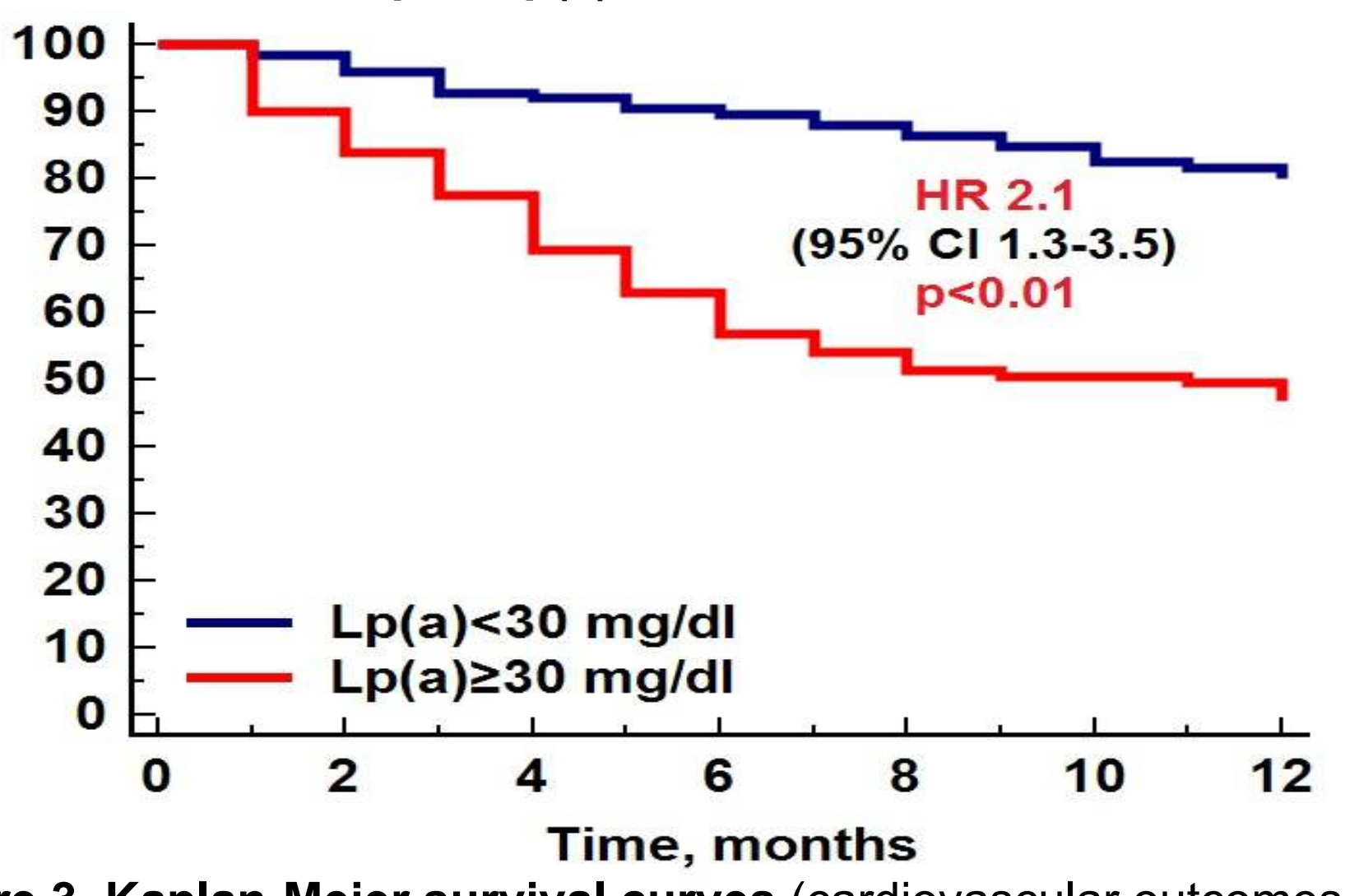

Figure 3. Kaplan-Meier survival curves (cardiovascular outcomes in 111 patients who underwent lower extremity revascularization depending on the $L p(a)$ level)
LEAD

Control

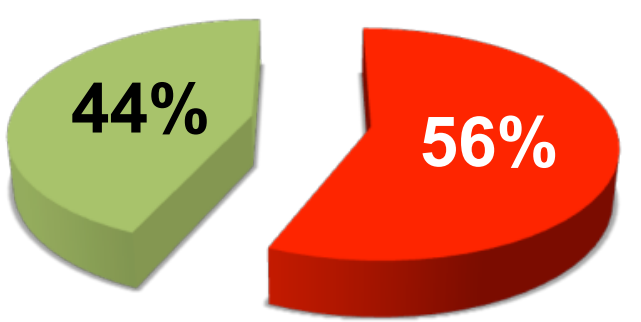

$p<0.001$

$\operatorname{Lp}(\mathrm{a})<30 \mathrm{mg} / \mathrm{dl}$

LEAD

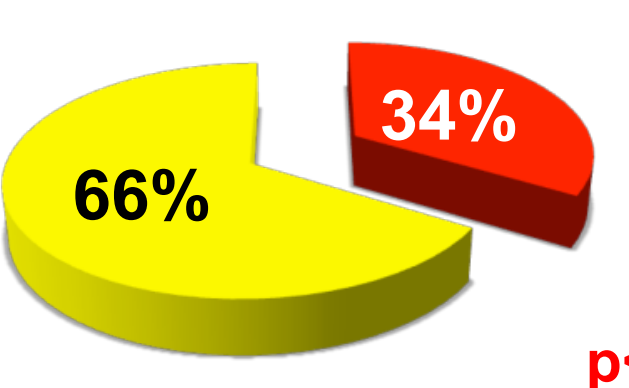

$p<0.001$

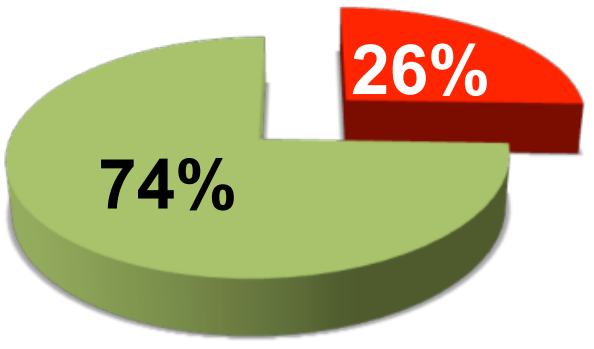

- $\mathrm{Lp}(\mathrm{a}) \geq 30 \mathrm{mg} / \mathrm{dl}$

Control

$\mathrm{Lp}(\mathrm{a})<50 \mathrm{mg} / \mathrm{dl}$

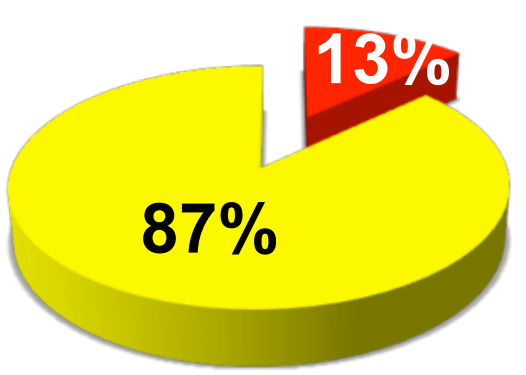

- $\mathrm{Lp}(\mathrm{a}) \geq 50 \mathrm{mg} / \mathrm{dl}$

Figure 1. Lp(a) excess in patients with LEAD and control group patients

Table 2. Logistic regression analysis of the risk of LEAD

\begin{tabular}{|l|c|c|}
\hline & OR & $95 \%$ Cl \\
\hline Age & $1.11^{*}$ & $1.08-1.14$ \\
\hline Male gender & $9.02^{*}$ & $5.09-15.96$ \\
\hline Hypertension & $2.55^{*}$ & $1.51-4.31$ \\
\hline Type 2 diabetes & $1.65^{*}$ & $1.24-2.19$ \\
\hline Smoking & $3.26^{*}$ & $2.00-5.33$ \\
\hline Lp(a) $\geq 30 \mathrm{mg} / \mathrm{dl}$ & $6.15^{*}$ & $3.20-11.80$ \\
\hline Lp(a) $\geq 50 \mathrm{mg} / \mathrm{dl}$ & $6.61^{*}$ & $3.13-13.96$ \\
\hline
\end{tabular}

\section{CONCLUSIONS}

Lp(a) level is an independent predictor of LEAD. Elevated Lp(a) is associated with two-fold increased risk of cardiovascular outcomes after lower extremity revascularization. of a Travel Grant for presenting author Narek Tmoyan 